\title{
Frustrated nematic order in spherical geometries
}

\author{
T. Lopez-Leon ${ }^{1}$, V. Koning ${ }^{2}$, K. B. S. Devaiah', ${ }^{1}$ V. Vitelli ${ }^{2}$ and A. Fernandez-Nieves ${ }^{1 \star}$
}

The prospect of mimicking molecular chemistry with colloidal rather than molecular building blocks could enable unprecedented control over the properties of microstructured materials'. The usual absence of directionality to the interaction between colloids has limited the complexity of the structures they can spontaneously form. One way to address this is to coat spherical colloid particles with a thin layer of nematic liquid crystal $^{2}$ and functionalize ${ }^{3}$ the unavoidable defects or bold spots that arise when nematic order is established on the surface of a sphere 4,5 . The number and arrangement of these defects can vary ${ }^{2,6-16}$, providing flexibility for tuning directional interactions that are more difficult to achieve by other methods ${ }^{17-26}$. Yet, many theoretically predicted structures have not been observed and control over defect location remains elusive. In this work, we show that varying the thickness of a nematic liquid crystal shell enables us to systematically control the number and orientation of defects formed. For thin shells, these defects can be engineered to emulate the linear, trigonal and tetrahedral geometries of $s p$, $s p^{2}$ and $s p^{3}$ carbon bonds, respectively. Such control opens up the possibility to engineer particles with tunable-valence and directional-binding capabilities.

To fabricate spherical nematic shells, we generate double emulsions with a microcapillary device ${ }^{27}$; these consist of a nematic drop that contains a smaller aqueous drop, all inside an aqueous continuous phase. Both the inner and outer water phases contain $1 \mathrm{wt} \%$ polyvinyl alcohol, which stabilizes the emulsion against coalescence and enforces tangential anchoring of the rod-like molecules of the nematic liquid crystal, pentylcyanobiphenyl. The resulting double-emulsion drops are characterized by an outer radius, $R$, of around $50 \mu \mathrm{m}$ and an inner radius, $a$, that are varied to produce shells of different average thicknesses, $\bar{h}=R-a$, as schematically shown in Fig. 1a. With this microfluidic method the thinnest shells that we can generate have $\bar{h} \approx 1 \mu \mathrm{m}$. However, it is possible to significantly reduce this value by increasing the volume of the inner drop once the double emulsion is formed. We achieve this by inducing a difference in osmotic pressure between the inner and outer water phases through the addition of a salt, $\mathrm{CaCl}_{2}$. As pentylcyanobiphenyl has a finite permeability to water, an incoming flow of water from the outer phase can be established if the inner drop contains a higher salt concentration than the outer phase. By controlling this difference, we can control the kinetics of the process and ultimately the thickness of the shells.

The thinnest shells have four defects, each with a topological charge $s=1 / 2$, reflecting the $\pi$ rotation experienced by the local nematic direction along a path encircling each defect. As a result, the total topological charge on the sphere is equal to $4 \times 1 / 2=2$; this is consistent with a mathematical theorem due to Poincaré and Hopf, which establishes that for nematic fields the total topological charge on a spherical surface must be equal to 2 . We image these shells using optical microscopy and change the focal plane of our microscope to precisely locate the position of the four defects; they are distributed throughout the shell, as shown by the images in Fig. 1b,c and by the schematic diagram in Fig. 1d. To obtain a meaningful value of the angular positions, we determine the position of all defects in a large number of shells with similar inner and outer drop sizes and plot the distribution for the central angle, $\theta$, which is the angle subtended by two defects with respect to the centre of the drop, and the distribution for the surface angle, $\alpha$, which is the angle subtended by two defects with respect to another defect. The resulting distributions are both Gaussian; they are centred at $\langle\theta\rangle=109^{\circ}$ and $\langle\alpha\rangle=60^{\circ}$ and have a width of $\Delta \theta=20^{\circ}$ and $\Delta \alpha=12^{\circ}$, as shown in Fig. 1e,f. The defects are thus located on the vertices of a tetrahedron.

Although this tetrahedral structure is predicted to be the ground state for nematic order on the surface of a sphere ${ }^{6}$, we observe two other coexisting configurations, which incorporate defects of charge $s=1$ (ref. 13). One of these structures is characterized by three defects, as shown in Fig. 1g,h and by the schematic diagram in Fig. 1i; two of them have $s=1 / 2$ and the other has $s=1$, adding up to a total of 2, consistent with the Poincaré-Hopf theorem ${ }^{4,5}$. The three defects form an isosceles triangle, where the unequal angle originates from the single $s=1$ defect, as schematically shown in Fig. 1j. The distribution for the two equal angles is centred at $\left\langle\alpha_{1}\right\rangle=68^{\circ}$ and has a width of $\Delta \alpha_{1}=15^{\circ}$, as shown in Fig. 1k, whereas the distribution of the unequal angle is centred at $\left\langle\alpha_{2}\right\rangle=46^{\circ}$ and has a width of $\Delta \alpha_{2}=12^{\circ}$, as shown in Fig. 1l. The other defect structure only possesses two $s=1$ defects, as shown in the images in Fig. 1m,n. The defects maximize their distance and align, on average, along the diameter of the drop, as schematically shown in Fig. 1o, and by the central angle distribution in Fig. 1p.

The defect arrangement in these very thin shells reflects the maximization of the mutual distance between defects expected for nematics on spherical surfaces. Indeed, the observed tetrahedral arrangement of four $s=1 / 2$ defects confirms the theoretical expectations for this situation ${ }^{6}$. The isosceles arrangement of the two $s=1 / 2$ defects and the $s=1$ defect is also consistent with our calculation of the equilibrium configuration for these three defects on a spherical surface, which corresponds to $\alpha_{1}=66^{\circ}$ and $\alpha_{2}=48^{\circ}$ (V. Koning \& V. Vitelli, unpublished results). For shells with two $s=1$ defects, the observed defect arrangement corresponds to the expected bipolar configuration, as any other configuration would lead to a smaller distance between defects. These results are the first experimental observations of these defect structures, expected for two-dimensional spherical nematics.

To address how these defect structures are affected by thickness, we minimize the elastic free energy:

$$
F=\frac{1}{2} K \int_{\text {shell }}\left(\nabla_{i} n_{j}\right)^{2} d^{3} r
$$



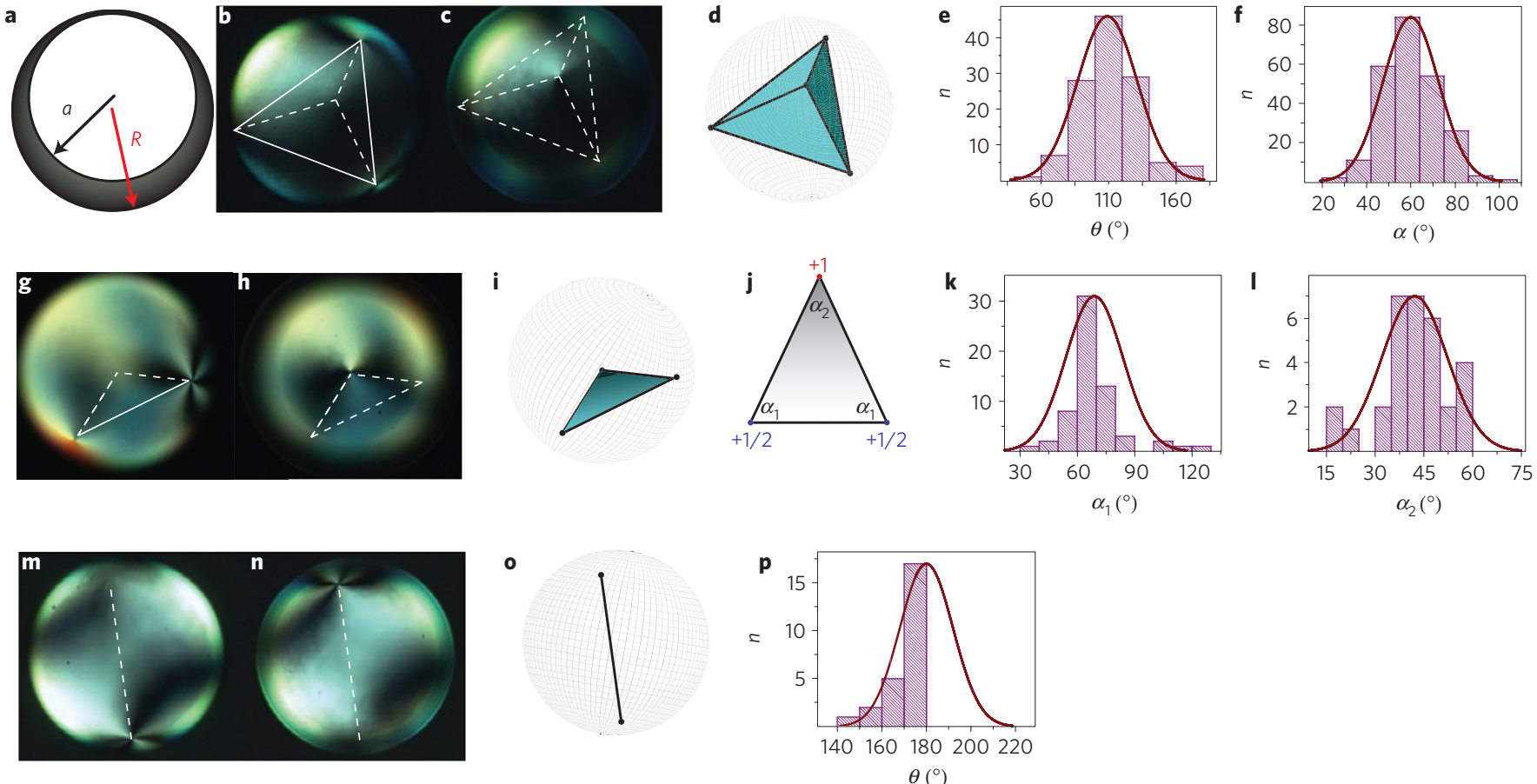

Figure 1 | Virtually two-dimensional shells. Defect configurations for very thin shells. a, Schematic diagram of a double emulsion drop with an inner drop of radius $a$ and a larger drop of radius $R$, displaced along the gravitational direction. $\mathbf{b}, \mathbf{c}$, Cross-polarized images of a very thin shell with four $s=1 / 2$ defects. $\ln \mathbf{b}$, two of the four defects are in focus, whereas in $\mathbf{c}$ we focus on the upper defect. $\mathbf{d}$, Schematic diagram of the observed arrangement of the four defects. e,f, Histograms for the central angle, $\theta$, and the surface angle, $\alpha$, between defects. g,h, Cross-polarized images of a very thin shell with three defects, two $s=1 / 2$ and an $s=1$. In $\mathbf{g}$, one of the three defects is clearly in focus, with another defect slightly below this one. In $\mathbf{h}$ the third defect is in focus. $\mathbf{i}$, Schematic diagram of the three-dimensional arrangement of the three defects. $\mathbf{j}$, Isosceles triangle formed by the three defects, with $\alpha_{2}$ the unequal surface angle. $\mathbf{k}, \mathbf{l}$, Histograms of the surface angles, $\alpha_{1}$ and $\alpha_{2}$. $\mathbf{m}, \mathbf{n}$, Cross-polarized images of a very thin shell with two $s=1$ defects. $\mathbf{0}$, Schematic diagram of the observed arrangement of the two defects. p. Histogram of the central angle, $\theta$.
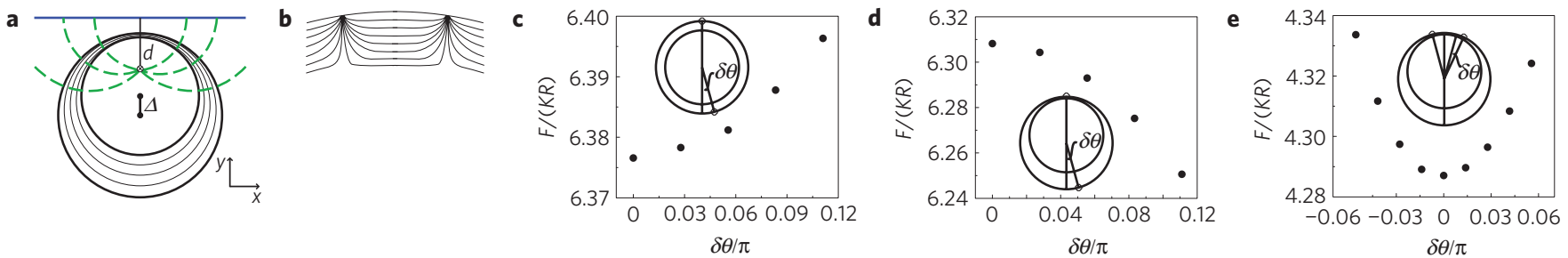

Figure 2 | Theoretical calculations. a, Equipotential (solid) and field (dashed) lines of an infinitely long charged wire, indicated with a cross, running along the $z$ direction, parallel to a conducting plate and at a distance $d$. The two equipotential circles, drawn in bold, correspond to two non-concentric drops whose centres, indicated by dots, are displaced by $\Delta . \mathbf{b}$, Slice of the nematic director in the thinner part of an inhomogeneous shell. c, Free energy of a shell of uniform thickness with $u=0.2$, as a function of the angular perturbation of one of the two pairs of defects, $\delta \theta$. When $\Delta=0$ the inner and outer drops are concentric and $\theta=\pi$ minimizes the free energy irrespective of thickness. $\mathbf{d}$, Free energy of a shell with $\Delta / \bar{h}=0.83$, as a function of $\delta \theta$. In this case, the $\theta=\pi$ arrangement no longer minimizes the free energy. e, Free energy as a function of the angular perturbation from the elastic-energy minimum, which is located at $\theta=30^{\circ}$ for the shell in $\mathbf{d}$.

expressed in terms of a single elastic constant, $K$, for the two-defect shell. We obtain an ansatz for the nematic director, $\mathbf{n}$, providing the local orientation of the rod-like molecules inside the shell, by analogy with the equipotential lines of an infinitely long charged wire located at a distance $d$ from a conducting plate. By choosing $d$ and two equipotential lines, we can fix the radii of the drops and their relative displacement from the concentric position, $\Delta$, as shown in Fig. 2a. Once the geometry of the boundary is fixed, we rotate the equipotential lines around the $y$ axis and independently position the line defects by choosing two electric-field lines, shown by dashed lines in Fig. 2a. We then carry out several rounds of numerical minimizations to ensure that each of the two line defects escapes in the third dimension, giving rise to two pairs of point defects on the bounding surfaces, as schematically shown in Fig. 2 b.
Using this director field, we can determine the central angle that minimizes the elastic free energy for different values of the relative thickness, $u=\bar{h} / R$, and of the normalized displacement, $\Delta / \bar{h}$. For concentric shells, $\Delta / \bar{h}=0$, and the energy minimum occurs for $\theta=180^{\circ}$, as shown in Fig. 2c, irrespective of thickness. By contrast, our analysis suggests that as $\Delta / \bar{h}$ is increased the antipodal arrangement of the pairs of defects can undergo an abrupt confinement transition to the thinnest part of the shell. In this case, the energy minimum at $\theta=180^{\circ}$ is lost, as shown in Fig. $2 \mathrm{~d}$, and pushed to much lower $\theta$. We confirm that this confined state corresponds to an elastic energy minimum by perturbing the angular separation between defects and seeing that the energy increases for larger and smaller angles, as shown in Fig. 2e. 

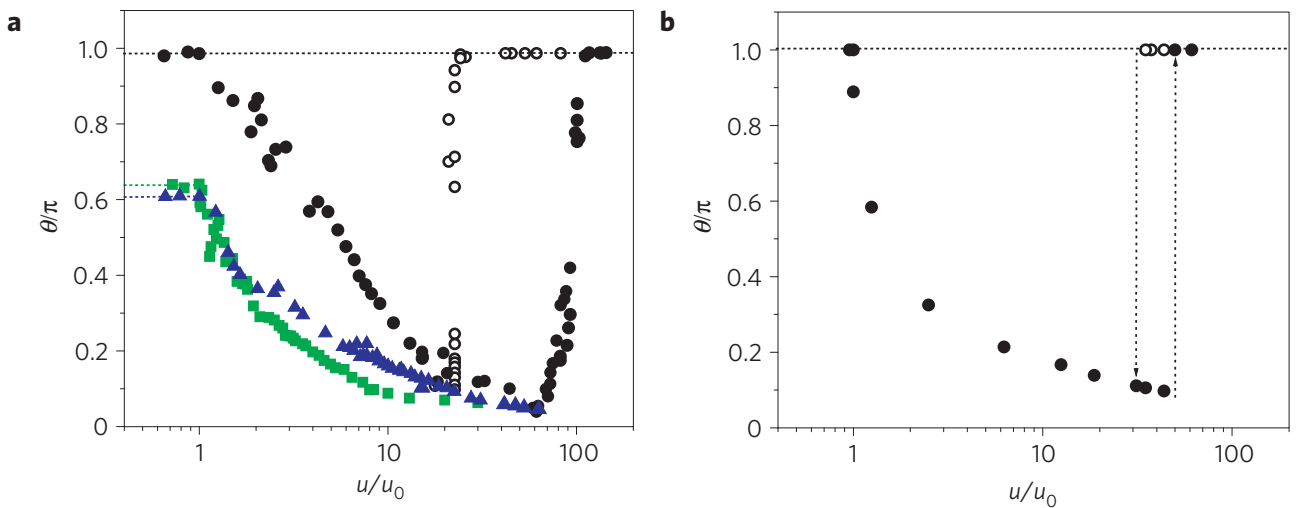

Figure 3 | Defect location versus thickness inhomogeneity. a, Experimental measurements of the central angle between two nearby $1 / 2$ defects (triangles) for the four-defect shells, a 1 and a 1/2 defect (squares) for the three-defect shells, and the two 1 defects (circles) for the two-defect shell, as a function of the normalized relative thickness, $u / u_{0}$, with $u=\bar{h} / R$ and $u_{0}$ the relative average thickness corresponding to the tetrahedral, isosceles and bipolar configurations characteristic of very thin shells. Open and filled circles illustrate the hysteresis of the discontinuous transition undergone by the defects in these shells. $\mathbf{b}$, Theoretical estimate of the central angle between two 1 defects at local free-energy minima as a function of $u / u_{0}$, while keeping $h_{1}$ constant. The confinement and deconfinement transitions occur at $u / u_{0} \sim 30$ and $u / u_{0} \sim 50$, respectively.

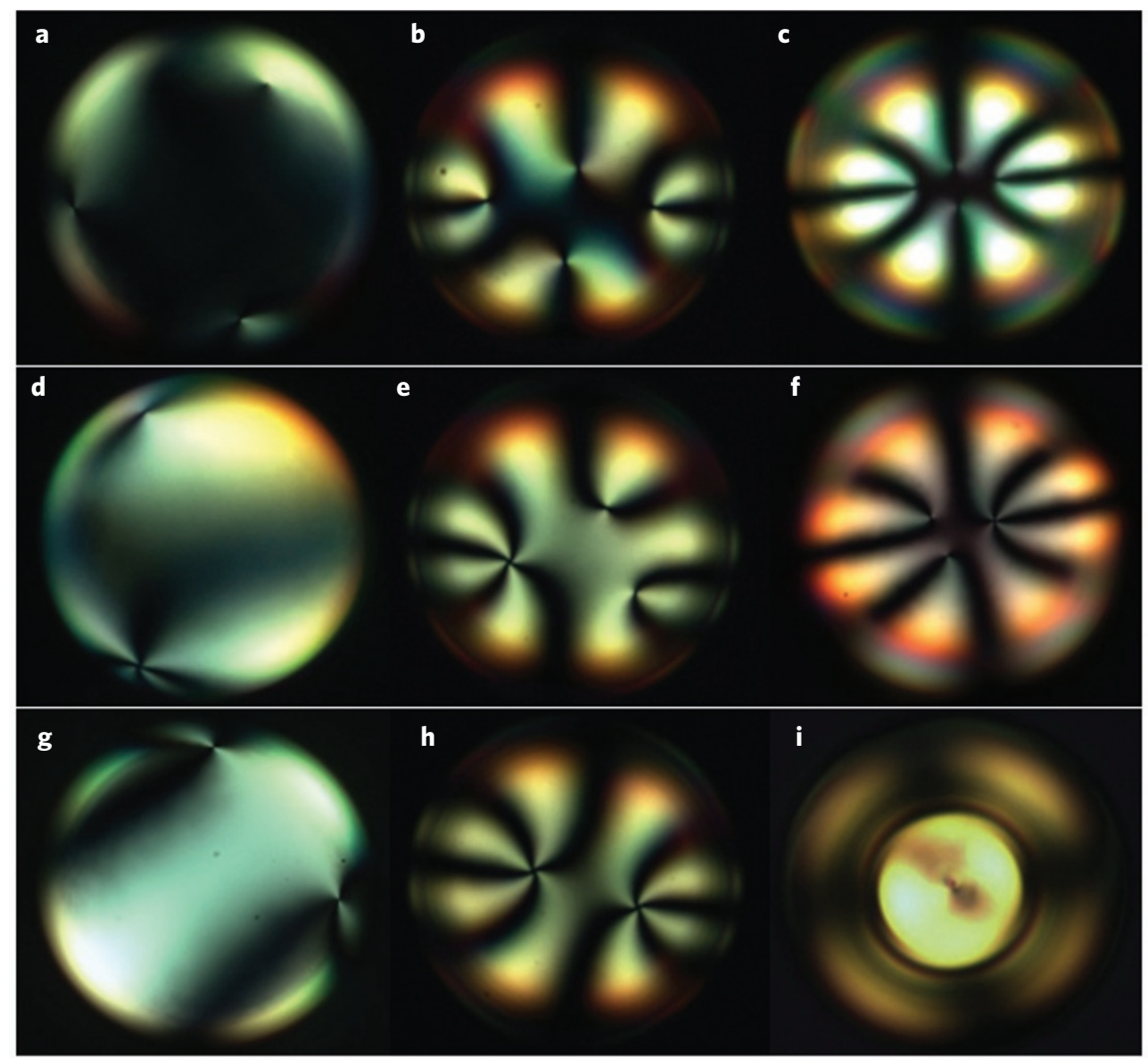

Figure 4 | Defect evolution with thickness inhomogeneity. a-i, Cross-polarized images illustrating the various angular configurations of the defects in shells with four defects $(\mathbf{a}-\mathbf{c})$, shells with three defects $(\mathbf{d}-\mathbf{f})$ and shells with two defects $(\mathbf{g}-\mathbf{i}) . \mathbf{a}, u / u_{0}=1.6 ; \mathbf{b}, u / u_{0}=7.8 ; \mathbf{c}, u / u_{0}=23.9 ; \mathbf{d}, u / u_{0}=1.2$; e, $u / u_{0}=4.6 ; \mathbf{f}, u / u_{0}=6.8 ; \mathbf{g}, u / u_{0}=7.1 ; \mathbf{h}, u / u_{0}=13.3 ; \mathbf{i}, u / u_{0}=53.7$.

The rich behaviour resulting from the thickness inhomogeneity can naturally be tested in our experiments by virtue of the density difference between the inner drop and the liquid crystal. Buoyancy displaces the inner drop out of the centre of the larger drop along the gravitational direction, as shown schematically in Fig. 1a. As a result, our shells have a thinnest region at the top, of thickness $h_{1}$, and a thickest region at the bottom, of thickness $h_{2}$. The value of $h_{1}$ remains essentially constant in our experiments, as it is determined by the steric repulsion between the two spherical interfaces in this region, which results from the presence of polyvinyl alcohol and the alignment of the pentylcyanobiphenyl molecules at these interfaces. As a result, by changing $a$ to change the average thickness of the shell, we change the value of $h_{2}$. This allows for controlled tuning of the shell-thickness inhomogeneity, which we achieve using osmosis. We note that the dynamic nature of the defects, which jiggle around their equilibrium position (see Supplementary Video), does not play a role in our experiments, because the timescale associated with this jiggling is many orders of magnitude smaller than the timescale associated with our osmotic experiments, which can thus be considered quasistatic. 
To quantify the shell thickness, we normalize $u$ with the relative shell thickness of the thickest shells where we observe the defect arrangements of Fig. 1; we denote this relative average thickness as $u_{0}$. For $u / u_{0} \leq 1$, the shells always exhibit the tetrahedral, isosceles or bipolar arrangement typical of nematics on spherical surfaces, as shown in Fig. 3, where we plot the central angle between two $s=1 / 2$ defects (triangles), an $s=1 / 2$ and the $s=1$ defect (squares) and the two $s=1$ defects (solid circles). Interestingly, for $u / u_{0}>1$, we observe a continuous change in the defect arrangement towards the thinnest part of the shell. As $u$ increases above $u_{0}$, the shells are made thicker and the thickness inhomogeneity increases, driving the spatial relocation of the defects, as shown in Fig. $4 \mathrm{a}-\mathrm{c}$ for a shell with four $s=1 / 2$ defects, in Fig. $4 \mathrm{~d}-\mathrm{f}$ for a shell with two $s=1 / 2$ defects and a $s=1$ defect and in Fig. 4g,h for a shell with two $s=1$ defects. In these cases, the $s=1 / 2$ defects are disclination lines spanning the shell, whereas each of the $s=1$ defect configurations is composed of two boojums, one residing on the inner surface and the other on the outer one ${ }^{13}$. For sufficiently large $u / u_{0}$, the defects are essentially confined to the thinnest part of the shell, with central angles that are much smaller than $180^{\circ}$, as shown in Fig. 3a.

Remarkably, for even larger $u / u_{0}$, we observe an abrupt transition in the defect location for two-defect shells. For $u / u_{0} \sim$ 80 , the defects rearrange themselves to maximize their separation, as shown in Fig. 3a with solid circles and in Fig. 4i, where we image the upper $s=1$ defect of a shell with the two pairs of boojums aligned vertically along the gravitational direction. The increased repulsion between defects can no longer be sustained and the shell transitions to a defect-deconfined state that maximizes the distance between them. A similarly abrupt transition happens if the process is reversed. In this case, we make double emulsions with a small drop containing a high salt concentration and allow the osmotic-pressure difference between the inner and continuous phases to drive solvent into the drop, which, as a result, progressively swells, making the shell thinner. We observe that the deconfined configuration of defects, with $\theta=180^{\circ}$, remains down to $u / u_{0} \sim 20$, where the defects abruptly become confined again to the thinnest part of the shell, as shown with open circles in Fig. 3a. For even lower $u / u_{0}$, the defects progressively spread through the shell to eventually maximize their separation when $u / u_{0}=1$. This scenario is in marked agreement with the results obtained from minimizing the elastic free energy for this geometry, as shown in Fig. $3 b$, where the angular separation of the two defects at the free-energy minima is plotted as a function of $u / u_{0}$ while keeping $h_{1}$ constant. Our elastic calculations suggest that the hysteresis in the abrupt transitions arises from the coexistence of confined and deconfined energy minima at large $u / u_{0}$ (V. Koning \& V. Vitelli, unpublished results).

Our results highlight the fascinating range of defect structures and defect arrangements that result from the interplay between the topological constraints imposed by the spherical geometry and the nematic order of liquid crystals. Although the tetrahedral structure originally predicted for nematics on spherical surfaces is indeed observed, it represents but one of a large number of other structures and configurations that arise from the thickness variation of the shells. This opens up a large spectrum of possibilities for inducing directional interactions between particles that are mediated by functionalized defects on their surfaces. Although this has been achieved for bipolar particles to create one-dimensional chains ${ }^{3}$, our results suggest that a wider range of structures, including the diamond structure, could emerge if the thickness inhomogeneity of the nematic coating is suitably tuned.

Received 12 August 2010; accepted 6 January 2011; published online 20 February 2011

\section{References}

1. Arsenault, A. et al. Towards the synthetic all-optical computer: Science fiction or reality? J. Mater. Chem. 14, 781-794 (2004).

2. Nelson, D. R. Toward a tetravalent chemistry of colloids. Nano Lett. 2, 1125-1129 (2002).

3. DeVries, G. A. et al. Divalent metal nanoparticles. Science 315, 358-361 (2007),

4. Hopf, H. Vektorfelder in $n$-dimensionalen Mannigfaltigkeiten. Math. Ann. 96, 427 (1926).

5. Poincaré, H. Mémoire sur les courbes définies par une équation différentielle. J. Math. Pures Appl. 1, 167 (1885).

6. Lubensky, T. C. \& Prost, J. Orientational order and vesicle shape. J. Phys. II 2, 371-382 (1992).

7. Dzubiella, J., Schmidt, M. \& Lowen, H. Topological defects in nematic droplets of hard spherocylinders. Phys. Rev. E 62, 5081-5091 (2000).

8. Bates, M. A. Nematic ordering and defects on the surface of a sphere: A Monte Carlo simulation study. J. Chem. Phys. 128, 104707 (2008).

9. Shin, H., Bowick, M. J. \& Xing, X. J. Topological defects in spherical nematics. Phys. Rev. Lett. 101, 037802 (2008).

10. Skacej, G. \& Zannoni, C. Controlling surface defect valence in colloids. Phys. Rev. Lett. 100, 197802 (2008).

11. Drzaic, P. S. Liquid Crystal Dispersions (World Scientific Publishing, 1995).

12. Kleman, M. \& Lavrentovich, O. D. Soft Matter Physics (Springer, 2001).

13. Fernandez-Nieves, A. et al. Novel defect structures in nematic liquid crystal shells. Phys. Rev. Lett. 99, 157801 (2007).

14. Vitelli, V. \& Nelson, D. R. Nematic textures in spherical shells. Phys. Rev. E 74, 021711 (2006).

15. Turner, A. M., Vitelli, V. \& Nelson, D. R. Vortices on curved surfaces. Rev. Mod. Phys. 82, 1301-1348 (2010).

16. Vitelli, V. \& Turner, A. M. Anomalous coupling between topological defects and curvature. Phys. Rev. Lett. 93, 215301 (2004).

17. Nagle, L. \& Fitzmaurice, D. Templated nanowire assembly on the surface of a patterned nanosphere. Adv. Mater. 15, 933-935 (2003).

18. Zhang, G., Wang, D. Y. \& Mohwald, H. Decoration of microspheres with gold nanodots—giving colloidal spheres valences. Angew. Chem. Int. Ed. 44, 7767-7770 (2005).

19. Cayre, O., Paunov, V. N. \& Velev, O. D. Fabrication of asymmetrically coated colloid particles by microcontact printing techniques. J. Mater. Chem. 13, 2445-2450 (2003).

20. Roh, K. H., Martin, D. C. \& Lahann, J. Biphasic Janus particles with nanoscale anisotropy. Nature Mater. 4, 759-763 (2005).

21. Li, F., Yoo, W. C., Beernink, M. B. \& Stein, A. Site-specific functionalization of anisotropic particles: From colloidal atoms to colloidal molecules. J. Am. Chem. Soc. 131, 18548-18555 (2009).

22. Glotzer, S. C. \& Solomon, M. J. Anisotropy of building blocks and their assembly into complex structures. Nature Mater. 6, 557-562 (2007).

23. Manoharan, V. N., Elsesser, M. T. \& Pine, D. J. Dense packing and symmetry in small clusters of microspheres. Science 301, 483-487 (2003).

24. Yin, Y. D., Lu, Y., Gates, B. \& Xia, Y. N. Template-assisted self-assembly: A practical route to complex aggregates of monodispersed colloids with well-defined sizes, shapes, and structures. J. Am. Chem. Soc. 123, 8718-8729 (2001).

25. Poulin, P., Stark, H., Lubensky, T. C. \& Weitz, D. A. Novel colloidal interactions in anisotropic fluids. Science 275, 1770-1773 (1997).

26. Musevic, I., Skarabot, M., Tkalec, U., Ravnik, M. \& Zumer, S. Two-dimensional nematic colloidal crystals self-assembled by topological defects. Science 313, 954-958 (2006)

27. Utada, A. S. et al. Monodisperse double emulsions generated from a microcapillary device. Science 308, 537-541 (2005).

\section{Acknowledgements}

We thank the National Science Foundation for CAREER award DMR-0847304. V.K. acknowledges funding from Stichting voor Fundamenteel Onderzoek der Materie (FOM). T.L-L. thanks the Marie-Curie programme.

\section{Author contributions}

T.L-L. carried out the experiments, analysed and interpreted the data and wrote the manuscript; V.K. carried out the theoretical calculations, analysed and interpreted the data and wrote the manuscript; K.B.S.D. carried out the experiments and analysed the data. V.V. supervised the theoretical work, interpreted the data and wrote the manuscript; A.F-N. supervised the experimental work, interpreted the data and wrote the manuscript.

\section{Additional information}

The authors declare no competing financial interests. Supplementary information accompanies this paper on www.nature.com/naturephysics. Reprints and permissions information is available online at http://npg.nature.com/reprintsandpermissions. Correspondence and requests for materials should be addressed to A.F-N. 